\title{
Utilização de recursos ópticos e equipamentos por escolares com deficiência visual
}

\author{
Utilization of optical devices and equipments by students with visual impairment
}

\author{
Rita de Cássia Ietto Montilha ${ }^{1}$ \\ Edméa Rita Temporini ${ }^{2}$ \\ Maria Inês Rubo de Souza Nobre ${ }^{3}$ \\ Maria Elisabete Rodrigues Freire Gasparetto ${ }^{4}$ \\ Newton Kara-Josés
}

\begin{tabular}{l} 
RESUMo \\
\hline Objetivo: Verificar percepções e conduta de escolares portadores de \\
deficiência visual, em relação aos recursos ópticos e equipamentos \\
utilizados no processo de escolarização. Métodos: Estudo descritivo \\
transversal em população de escolares de 12 anos e mais, portadores de \\
deficiência visual, congênita ou adquirida, em processo de escolarização, \\
inseridos no sistema público de ensino no município de Campinas no ano \\
de 2000. Aplicou-se questionário por entrevista, elaborado com base em \\
estudo exploratório. Resultados: Foi obtida população de 26 alunos, \\
sendo 46,2\% portadores de visão subnormal e 53,8\% portadores de \\
cegueira. A maioria cursava o ensino fundamental (65,4\%) em escolas \\
com salas de recursos (73,1\%). Entre os recursos utilizados em atividades \\
de leitura e escrita 94,1\% dos escolares declararam utilizar a máquina \\
Braille e $81,8 \%$ relataram que o colega dita a matéria. A maioria dos \\
escolares com visão subnormal utilizavam óculos (91,7\%) e 33,3\% utiliza- \\
vam lupa como recursos ópticos. Entre os recursos não ópticos desta- \\
caram-se os ambientais - aproximação da lousa (75,0\%) e da janela \\
(66,7\%\%) para maior iluminação. Conclusões: Foi evidenciado o fato de \\
que escolares portadores de visão subnormal utilizavam recursos des- \\
tinados a portadores de cegueira como a aplicação do sistema Braille. \\
Verificou-se reduzido número de portadores de visão subnormal utilizan- \\
do recursos ópticos e não ópticos referentes à sua problemática, o que \\
indica provável desconhecimento de seu potencial visual e de recursos \\
apropriados para melhorar sua eficiência.
\end{tabular}

Descritores: Recursos audiovisuais; Leitura; Baixa visão; Portadores de deficiência visual; Cegueira/prevenção \& controle; Estudantes

\section{INTRODUÇÃO}

Esta pesquisa versa sobre escolares portadores de deficiência visual, inseridos no sistema público de ensino no município de Campinas, São Paulo, suas percepções e conduta em relação ao próprio processo de escolarização.

Adota-se como conceito de deficiência visual a presença de cegueira ou visão subnormal. Segundo a Organização Mundial de Saúde (OMS), é considerado portador de cegueira o indivíduo com acuidade visual desde 3/60 (0,05), no melhor olho e melhor correção óptica possível, até ausência de percepção de luz, ou correspondente perda de campo visual no melhor olho com a melhor correção possível ${ }^{(1)}$. A definição de visão subnormal corresponde à acuidade visual igual ou menor do que 6/18 (0,3) mas, igual ou maior do que 3/60 $(0,05)$ no melhor olho com a melhor correção possível. 
Visão subnormal (VSN) é definida também como uma perda acentuada da visão que não pode ser corrigida por tratamento clínico ou cirúrgico, nem com óculos convencionais. Também pode ser descrita como qualquer grau de enfraquecimento visual que cause incapacidade funcional e diminua o desempenho visual ${ }^{(2)}$.

Os profissionais que atuam na reabilitação e educação de indivíduos deficientes visuais necessitam deter conhecimentos sobre as limitações desses indivíduos bem como sobre o sistema de ensino e reabilitação vigentes. A escola e a reabilitação devem caminhar juntas, suprindo as reais dificuldades da criança, do adolescente e do adulto portador de deficiência visual.

Reconhece-se no mundo todo a necessidade de identificar indivíduos portadores de afecções oculares. O dimensionamento de aspectos dessa problemática, por meio da investigação científica, provê base concreta para o planejamento de ações curativas e preventivas relacionadas à saúde ocular.

Em termos de Saúde Pública destacam-se três campos principais a merecerem investigação: os que compreendem a variável técnica ou tecnológica, a variável administrativa e a variável humana ou social ${ }^{(3)}$.

"O planejamento de ações de saúde que requerem a participação da comunidade deve ser realizado mediante o conhecimento prévio acerca do que aquelas pessoas sabem, acreditam, desejam ou rejeitam e fazem, em relação ao problema de saúde, objeto do programa"(3-4). Esse princípio é aplicável também às ações educativas.

Nesse enfoque, a metodologia deste estudo refere-se à pesquisa de fatores humanos.

Segundo a OMS a avaliação multidisciplinar envolvendo médicos, psicólogos e educadores levam à compreensão das necessidades educacionais especiais da criança ${ }^{(5)}$. Estes novos conceitos associados à melhor compreensão das conseqüências psicológicas e educacionais da deficiência visual aumentam as oportunidades dessas crianças, mesmo as portadoras de níveis de visão quantitativamente baixos. Programas foram desenvolvidos não apenas para avaliar os níveis de percepção visual, como também para valorizar as informações clínicas sobre acuidade e campo visual.

A sociedade arca com prejuízos elevados em decorrência da falta de atenção com a saúde visual, representados pela diminuição de produtividade da sua força de trabalho e o elevado custo de ações de reabilitação. Acrescem-se a isto, conseqüências psicológicas, sociais e econômicas para o deficiente visual devidas às restrições ocupacionais, diminuição da renda, perda de "status", de auto-estima, de autoconfiança. Desse modo, a qualidade de vida é afetada, como vem-se observando especialmente em países em desenvolvimento ${ }^{(6-8)}$.

Nesse sentido observa-se a necessidade de conhecer melhor a situação dos deficientes visuais para que haja o planejamento de ações que minimizem tais prejuízos.

A criança/adolescente, portadora de deficiência visual está inserida no sistema público de ensino? Ela conhece e utiliza os recursos apropriados às suas necessidades para viabilizar seu processo de escolarização?
Essas indagações deram origem ao presente estudo, que teve o objetivo de verificar percepção e conduta de escolares portadores de deficiência visual em relação aos recursos ópticos e equipamentos utilizados no processo de escolarização.

\section{MÉTODOS}

Realizou-se um "survey" descritivo, tipo transversal.

A população foi composta por escolares de 12 anos e mais, portadores de deficiência visual, inseridos no sistema público de ensino no município de Campinas, no ano de 2000. A população foi obtida por indicação do Serviço de Educação Especial da Prefeitura Municipal de Campinas, por professores especialistas, professores de salas de recursos, professores de salas da Fundação Municipal de Educação Comunitária (FUMEC) - Programa de jovens e adultos; por informações contidas no cadastro dos serviços de reabilitação de deficientes visuais do município de Campinas e do Serviço de Visão Subnormal da UNICAMP. A escolha desse grupo etário, delimitando as características da população, deveu-se ao fato de ser esta a idade mínima considerada necessária para que os escolares forneçam informações em entrevista.

Foi realizado estudo exploratório que permitiu conhecer previamente a realidade e a terminologia utilizada por escolares com as características definidas para a população do estudo, residentes em outros municípios da região, próximos ao município de Campinas.

A partir da análise das entrevistas realizadas no estudo exploratório, foi elaborado um questionário adequado à realidade investigada, tendo sido submetido a teste prévio. O teste prévio também foi aplicado a escolares de outros municípios. A coleta de dados foi realizada de agosto a dezembro de 2000, aplicando-se o questionário por entrevista.

\section{RESULTADOS}

A população de escolares foi composta por 26 alunos portadores de deficiência visual, sendo $46,2 \%$ portadores de visão subnormal e 53,8\% portadores de cegueira.

Os alunos manifestam dúvidas em relação ao próprio grau de dificuldade para escolarização $(46,2 \%)$ e 30,8\% declaram difícil escolarização (Tabela 1).

Entre os recursos utilizados para atividades de leitura e escrita, 94,1\% dos escolares recorrem à máquina Braille e $81,8 \%$, ao colega que dita a matéria (Tabela 2 ).

\begin{tabular}{|c|c|c|}
\hline Grau de dificuldade & $f$ & $\%$ \\
\hline Fácil & 8 & 30,8 \\
\hline Nem fácil, nem difícil & 12 & 46,2 \\
\hline Difícil / Não tem opinião & 6 & 23,0 \\
\hline
\end{tabular}




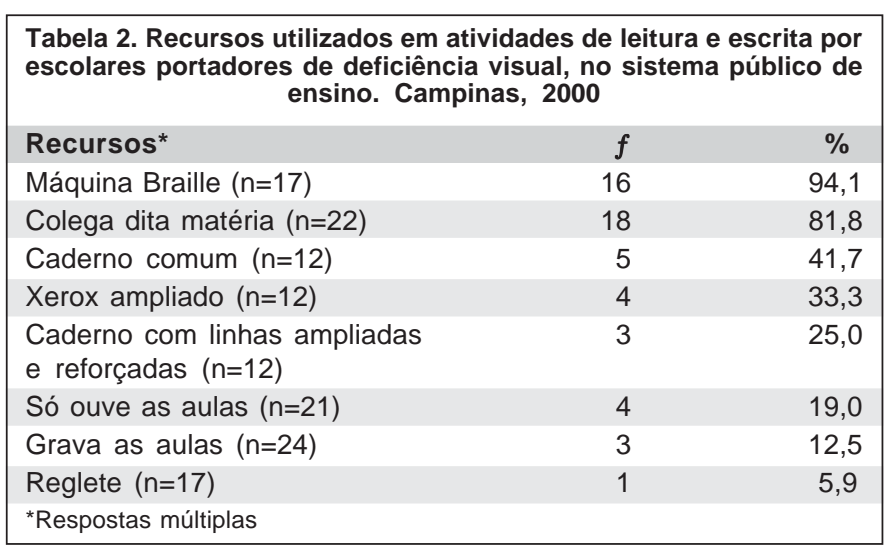

A maioria de escolares portadores de deficiência visual estuda em escola que conta com sala de recursos $(73,1 \%)$ (Tabela 3). Os demais buscam orientações nas salas de recursos, mesmo não situando na escola que freqüentam. Nenhum recebe orientação de professor itinerante.

Em relação à escolaridade, a maioria encontra-se cursando o ensino fundamental $(65,4 \%)$.

A maioria dos escolares portadores de visão subnormal utilizam óculos $(91,7 \%)$ e 33,3\% utilizam lupa como recursos ópticos. No que se refere aos recursos não ópticos destacamse os ambientais - aproximação da lousa e da janela para maior iluminação, 75,0\% e 66,7\% respectivamente (Tabela 4).

Os escolares revelam-se muito satisfeitos com o trabalho do professor (88,5\%), (Tabela 5).

\section{DISCUSSÃO}

O conhecimento de percepções de escolares portadores de deficiência visual a respeito de seu processo de escolarização, é fundamental para o planejamento de ações educativas para essa população.

No Brasil, tem sido ressaltada a necessidade de identificação do número de portadores de deficiência visual. Neste estudo, registrou-se população reduzida de portadores de deficiência visual (26 escolares). Imaginava-se uma população maior de escolares com deficiência visual inseridos no ensino público. Segundo alguns autores foram identificados 10 escolares portadores de visão subnormal na triagem oftalmológica da campanha comunitária de reabilitação visual na escola, realizada em 1999, em Campinas ${ }^{(9)}$. Esses escolares cursavam a $1^{\text {a }}$ série do ensino fundamental no sistema público do município. É admissível imaginar que, se a cada ano, dez alunos portadores de visão subnormal iniciam o processo de escolarização o número de alunos com visão subnormal somado aos que apresentam cegueira, considerando ensino fundamental e médio deveria ser maior. Pode-se supor que muitos deles tenham tido dificuldades de ingresso à escola ou no decorrer dos primeiros anos de atividades escolares.

Segundo a OMS mais de $90 \%$ das pessoas deficientes visuais no mundo devem estar distribuídas nos países em desenvolvimento, a maioria localizada na Ásia em comunidades rurais ${ }^{(5)}$. A ausência de estatísticas precisas nesses países dificulta a compreensão da problemática. Estima-se que crianças com baixa visão sejam matriculadas no sistema público de ensino como videntes, sendo simplesmente absorvidas pelo sistema educacional. Pais e professores nem sempre percebem que a criança apresenta um problema visual. Devido a sua dificuldade tornam-se crianças de "alto risco" e abandonam as escolas. Talvez esse seja um dos motivos de, neste estudo, a proporção de escolares com visão subnormal ter sido menor do que a de portadores de cegueira.

Escolares portadores de visão subnormal e não apenas os

\begin{tabular}{|c|c|c|}
\hline Sala de recursos & $f$ & $\%$ \\
\hline Sim & 19 & 73,1 \\
\hline Não & 7 & 26,9 \\
\hline
\end{tabular}

\begin{tabular}{|c|c|c|}
\hline Recursos ópticos e não ópticos* & $f$ & $\%$ \\
\hline Utiliza óculos & 11 & 91,7 \\
\hline Senta próximo à lousa & 9 & 75,0 \\
\hline Senta perto da janela & 8 & 66,7 \\
\hline Utiliza lápis com grafite mais escuro & 7 & 58,3 \\
\hline Utiliza caderno com linhas reforçadas & 4 & 33,3 \\
\hline Utiliza lupa & 4 & 33,3 \\
\hline Utiliza caderno com linhas espassadas & 3 & 25,0 \\
\hline Utiliza Braille & 3 & 25,0 \\
\hline Coloca iluminação dirigida ao material de leitura & 2 & 16,7 \\
\hline Utiliza caneta com ponta porosa & 2 & 16,7 \\
\hline
\end{tabular}

Tabela 5. Grau de satisfação de escolares portadores de deficiência visual, em relação ao ambiente escolar, material utilizado, professores e direção da escola. Campinas, $2000(\mathrm{n}=26)$

\begin{tabular}{|c|c|c|c|c|c|c|}
\hline \multirow[t]{2}{*}{ Grau de satisfação } & \multicolumn{2}{|c|}{ Materiais e ambiente escolar } & \multicolumn{2}{|c|}{ Professores } & \multicolumn{2}{|c|}{ Diretor(a) } \\
\hline & $f$ & $\%$ & $f$ & $\%$ & $f$ & $\%$ \\
\hline Muito satisfeito & 9 & 34,6 & 23 & 88,5 & 12 & 46,2 \\
\hline Mais ou menos satisfeito & 11 & 42,3 & 2 & 7,7 & 14 & 53,8 \\
\hline Pouco / Nada satisfeito & 6 & 23,1 & 1 & 3,8 & - & - \\
\hline
\end{tabular}


portadores de cegueira utilizam, como recursos para atividades de leitura e escrita na escola, o sistema Braille $(94,1 \%)$ e recorrem ao colega que dita a matéria $(81,8 \%)$ (Tabela 2$)$. Este fato reforça a idéia de que alunos com baixa visão podem desconhecer seus próprios recursos para a execução dessas atividades, como o uso de auxílios ópticos, textos com tipos ampliados e outros recursos não ópticos que poderiam facilitar seu processo de escolarização ${ }^{(10-12)}$.

Isto pode relacionar-se ao fato de que os professores, familiares e o próprio escolar desconhecem o potencial visual do portador de visão subnormal e os recursos ópticos e não ópticos que podem beneficiá-lo na execução de atividades escolares. Nem o próprio aluno nem o seu professor conhecem, muitas vezes, os recursos ambientais que podem favorecer o aprendizado do aluno de baixa visão (sentar-se próximo à lousa e próximo à janela para melhor iluminação, entre outros). É importante ressaltar a orientação à família e também a capacitação do professor no sentido de conhecer os recursos e estimular o aluno a usá-los.

Alguns autores recomendam que o aluno com visão subnormal utilize alguns dispositivos para favorecer o funcionamento e eficiência visual, entre eles, canetas tipo pincel atômico, acetato, papel com pautas em negrito e outros; as autoras ressaltam que os materiais não precisam ser prescritos por um especialista, mas os professores necessitam saber as razões da utilização desses recursos ${ }^{(12)}$.

Fatores psicológicos podem estar relacionados a não utilização de auxílios ópticos e não ópticos. Sabe-se que, principalmente na adolescência, a aceitação do grupo é fator determinante do comportamento de jovens. Usar um recurso óptico diferente dos óculos comuns, que não apresente uma estética desejável, ou mesmo o material escolar diferente dos demais colegas, pode causar problemas de auto-estima e de conseqüente aceitação da condição de portador de deficiência.

Estudo realizado no SVSN - UNICAMP em 1998, realizou reavaliação de 90 escolares que haviam sido atendidos no serviço. A pesquisa mostrou que $89,7 \%$ dos escolares utilizavam o auxílio óptico na escola e/ou em casa, considerada uma adesão satisfatória. Dos 14,4\% que não estavam fazendo uso efetivo, a metade referiu-se a problemas de rejeição por motivos estéticos, a outra metade dividiu-se igualmente entre dificuldades financeiras em adquirir o auxílio, diminuição da acuidade visual e prescrição inadequada ${ }^{(13)}$.

A maioria dos escolares entrevistados estuda em escola com "sala de recursos" (73,1\%) (Tabela 3). Sala de recursos é definida como local com equipamentos, materiais e recursos pedagógicos específicos à natureza das necessidades especiais do educando, onde se oferece a complementação do atendimento educacional realizado em classes do ensino comum, por professor especializado. São definidos para a sua implementação aspectos físicos e pedagógicos específicos ${ }^{(14)}$.

São várias as atribuições da "sala de recursos", entre elas destacam-se: apoiar a integração do aluno portador de deficiência visual na escola e na comunidade escolar; prestar assessoramento técnico-pedagógico aos professores da rede regular de ensino para a elaboração de um plano de atendimento adequado às necessidade individuais do aluno portador de deficiência visual; fornecer material didático especializado ou adaptado, necessário ao desenvolvimento do currículo; garantir o suprimento de material de transcrição Braille/tinta, tinta/ Braille, ampliação, gravação sonora de textos, adaptação de gráficos, mapas e similares, além de materiais didáticos para uso de cegos e portadores de visão subnormal ${ }^{(14-15)}$.

Considerando as atividades desenvolvidas nas salas de recursos, justifica-se o fato de que a maioria dos escolares optaram pelas escolas que sediam tal recurso. Os escolares, que freqüentam escolas que não dispõem da referida sala, buscam este recurso no período inverso ao das aulas para desenvolverem atividades específicas, para transcrição de textos e trabalhos. Os professores desses alunos também recorrem às salas de recursos para solicitarem transcrição de provas. Observando-se que os escolares entrevistados dividem-se entre 3 salas de recursos, pode-se supor um acúmulo de tarefas para esses professores especializados.

Os alunos relataram também que recorrem à ajuda de familiares para a transcrição do material em Braille, com a finalidade de agilizar o acesso ao material. Tal fato sugere que a demora do acesso ao material didático possa prejudicar o desempenho escolar dos alunos com deficiência visual.

Recursos ópticos foram mencionados por apenas 33,3\% (lupa) dos portadores de visão subnormal, além dos óculos comuns $(91,7 \%)$ (Tabela 4). Não é possível avaliar se mais alunos precisariam de outros recursos ópticos, mas a questão merece destaque, se observada a melhora de funcionalidade visual com a utilização de recursos apropriados. Em relação aos recursos não ópticos destacaram-se os ambientais - aproximação da lousa e da janela para maior iluminação (Tabela 4).

Recentes estudos mostram que, com a utilização de auxílio ópticos prescritos de acordo com o nível visual apresentado pelo sujeito, a funcionalidade visual pode ser melhorada. Estudo realizado na Inglaterra evidencia o acompanhamento de 168 pacientes portadores de visão subnormal que tiveram prescrição de auxílio ópticos associados a orientação de uso de recursos não ópticos e foram acompanhados por seis meses consecutivos. No que se refere à atividade de leitura melhoraram o grau de desempenho significativamente, $88,0 \%$ dos pacientes começaram a ler letras impressas em jornais depois do tratamento mencionado ${ }^{(10)}$.

Estudo realizado em Campinas mostrou a adesão de 85,6\% de escolares atendidos no serviço de visão subnormal do Hospital de Clínicas da UNICAMP, ao uso do auxílio óptico após o tratamento no referido serviço. Nesse estudo foram realizados esclarecimentos aos professores e familiares sobre os benefícios do uso dos auxílios ópticos ${ }^{(13)}$.

$\mathrm{O}$ fato dos escolares mostrarem-se muito satisfeitos com o trabalho do professor $(88,5 \%)$, porém pouco ou nada satisfeitos com materiais e ambiente escolar $(23,1 \%)$, sugere que embora reconheçam a existência de recursos, eles não têm acesso a eles (Tabela 5).

Observa-se que o cliente portador de VSN só apresenta um bom aproveitamento das orientações realizadas, das experiências vividas com os materiais, das adaptações e auxílios ópti- 
cos, se houver conscientização de suas habilidades, limitações e boa auto-estima ${ }^{(16)}$.

$\mathrm{Na}$ última década verifica-se a efetivação de legislações, políticas e declarações nacionais e internacionais voltadas para a educação inclusivista de pessoas com necessidades especiais, porém, na prática, verifica-se ainda grande discrepância em relação ao que dizem as leis, os professores e os alunos ${ }^{(17-20)}$.

Essas mudanças requerem a capacitação de professores que devem contar com conhecimentos especializados. Apoios informais e formais essenciais às comunidades inclusivas, que respondem as necessidades de todos os alunos, podem ser facilitados suprimindo-se as escolas e classes especiais. Os professores das escolas especiais podem tornar-se professores regulares, professores de equipe, especialistas e colaboradores em recursos humanos e facilitadores de redes de apoio na educação regular. Além disso, a riqueza de materiais, procedimentos, apoios, equipamentos e recursos da educação especial podem ser integrados à educação geral ${ }^{(14-15,19)}$.

Outro importante elemento no desenvolvimento da rede de apoio e da comunidade inclusiva é a oportunidade de intercâmbio proporcionado pelas diferenças individuais. Ela fortalece a escola e a sala de aula e oferece a todos os seus membros maiores oportunidades de aprendizagem ${ }^{(19-20)}$.

\section{CONCLUSÕES}

Em relação às percepções sobre o processo de escolarização os escolares manifestaram dúvida referente ao próprio grau de dificuldade para a escolarização.

No que se refere à conduta, entre os escolares portadores de visão subnormal poucos declararam utilizar auxílios ópticos, alguns mencionaram utilizar o sistema Braille como recurso para atividades escolares, sugerindo, talvez, o desconhecimento de recursos ópticos apropriados à sua condição visual ou até mesmo de seu potencial visual. Já entre os portadores de cegueira o uso da máquina Braille destacou-se.

\section{ABSTRACT}

Purpose: To verify perceptions and conduct of students with visual impairment regarding devices and equipment utilized in schooling process. Methods: A transversal descriptive study on a population of 12-year-old or older students in schooling process, affected by congenital or acquired visual impairment, inserted in the government teaching system of Campinas during the year 2000. An interview quiz, created based on an exploratory study was applied. Results: A group of 26 students, $46 \%$ of them with low vision and $53.8 \%$ affected by blindness was obtained. Most of the students were from fundamental teaching courses (65.4\%), studying in schools with classrooms provided with devices (73.1\%). Among the resources used in reading and writing activities, $94.1 \%$ of the students reported they used the Braille system and $81.8 \%$ reported that the reading subject was dictated by a colleague. Most of the students with low vision wore glasses (91.7\%), and $33.3 \%$ utilized a magnifying glass as optical devices. Among the non-optical devices, the most common were the environmental ones, getting closer to the blackboard $(75.0 \%)$ and to the window $(66.7 \%)$ for better lighting. Conclusions: It became evident that students with low vision eye-sight made use of devices meant for bearers of blindness, such as applying the Braille system. A reduced number of low vision students making use of optical and non-optical devices applicable to their problems were observed, indicating a probable unawareness of their visual potential and the appropriate devices to improve efficiency.

Keywords: Audiovisual aids; Reading; Vision, low; Visually impared persons; Blindness/prevention \& control; Students

\section{REFERÊNCIAS}

1. Thylefors B, Negrel AD, Pararajasegaram R, Dadzie KY. Global data on blindeness. Bull World Health Organ. 1995;73(1):115-21.

2. Carvalho KMM, Gasparetto MERF, Venturini NHB, Melo HFR. Visão subnormal: orientações ao professor do ensino regular. 2a ed. Campinas: Editora da UNICAMP; 1994.

3. Temporini ER. Pesquisa de oftalmologia em Saúde Pública: considerações metodológicas sobre fatores humanos. Arq Bras Oftalmol. 1991;54(6):279-81.

4. Piovesan A, Temporini ER. Pesquisa exploratória: procedimento metodológico para o estudo de fatores humanos no campo da saúde pública. Rev Saúde Pública. 1995;29(4):318-25.

5. WHO Programme for the Prevention of Blindness. Management of low vision in children: report of a WHO consultation, Bangkok, 23-24 July 1992. Geneva: World Health Organization; 1992.

6. World Health Organization. Social and behavioural aspects of comprehensive eye care: report. Brussels: Regional Office for Europe; 1994.

7. TemporinI ER, Kara-José N. Níveis de prevenção de problemas oftalmológicos: propostas de intervenção. Arq Bras Oftalmol. 1995;58(3):189-94.

8. Kara-José N, Temporini ER. Cirurgia de catarata: o porquê dos excluídos. Rev Panam Salud Publica. 1999;6(4):242-8.

9. Nobre MIRS, Temporini ER, Kara-José N, Montilha RCI. Deficiência visual de escolares: percepções de mães. Temas Desenvolv. 2001;10(55):24-7.

10. Margrain TH. Helping blind and partially sighted people to read: the effectiveness of low vision aids. Br J Ophthalmol. 2000;84(8):919-21.

11. Stochholm K. Utopia e realidade: trinta anos de educação integrada. Rev Benjamin Constant. 1997;6:3-6.

12. Torres SI, Corn AL. When you have a visually handicapped child in your classroom: sugestions for teachers. 2th ed. New York: American Foundation for the Blind; 1990.

13. Brasil. Ministério da Saúde. Coordenação de Atenção a Grupos Especiais. Programa de Atenção à Saúde da Pessoa Portadora de Deficiência. Atenção à pessoa portadora de deficiência no Sistema Único de Saúde: planejamento e organização de serviços. Brasília: Secretaria de Assistência à Saúde; 1993. 48p.

14. Brasil. Ministério da Educação e do Desporto. Secretaria de Educação Especial. Subsídios para organização e funcionamento de serviços de educação especial: área de deficiência visual. Brasília: MEC/SEESP; 1995. 58p. (Série Diretrizes, 8).

15. Cerqueira JB, Ferreira EMB. Recursos didáticos na educação especial. Rev Benjamin Constant. 2000;15:24-8.

16. Montilha RCI. Visão subnormal e a abordagem da terapia ocupacional. Sinopse Oftalmol. 2001;3(1):22-4.

17. Azevedo TFP. Educação da pessoa com necessidades especiais: o caso de Juiz de Fora. Temas Desenvolv. 2000;9(50):40-7.

18. Masini EFS. A educação do portador de deficiência visual - as perspectivas do vidente e do não vidente. In: Alencar EML. Tendências e desafios da educação especial. Brasília, SEESP; 1994. p.103.

19. Veríssimo H. Inclusão: a educação da pessoa com necessidades educativas especiais: velhos e novos paradigmas. Rev Benjamin Constant. 2001;18:6-10.

20. Bruno MMG. Educação inclusiva: problemas e perspectivas. In: $2^{\circ}$ Encontro de Educação do Oeste Paulista. Políticas Públicas: Diretrizes e necessidades da Educação Básica. UNESP; Anais. Presidente Prudente (SP); 2000. p.79-90. 